\title{
Grünes Licht für herzinsuffiziente Kaffeefreunde
}

\author{
Entwarnung für Koffeinjünger: Beim Kaffeekränzchen dürfen Patienten mit Herzinsuffizienz \\ die ganze Kanne austrinken, ohne mit kardialen Unannehmlichkeiten rechnen zu müssen.
}

\begin{abstract}
_ Für eine doppelblinde Cross-overStudie hielten 51 Patienten mit moderater bis schwerer Herzinsuffizienz (NYHA-Klassen I-III, Ejektionsfraktion der linken Herzkammer < 45\%) zunächst sieben Tage Koffeinkarenz. Danach wurde ihnen randomisiert entweder 500 mg Koffein oder Laktosepuder verabreicht, aufgelöst in fünf Tassen entkoffeniertem Kaffee im Abstand von je einer Stunde. Es erfolgten eine kontinuierliche EKG-Ableitung (im Mittel über 6,7 Stunden), Blutentnahmen vor und nach sowie ein Belastungs-EKG nach Kaffeekonsum. Nach einer einwöchigen Auswaschphase folgte das Cross-over.

Zwischen Koffein- und Placebogruppe gab es keine signifikanten Unter-
\end{abstract}

schiede hinsichtlich ventrikulärer (185 vs. 239) und supraventrikulärer Extrasystolen pro Stunde, Couplets, Bigeminie oder nicht-anhaltender Tachykardien. Auch beim Belastungs-EKG zeigten sich keine Unterschiede, auch nicht bei Patienten mit niedrigen Plasmakoffeinspiegeln von $5.200 \mu \mathrm{g} / \mathrm{l}$ oder hohen Spiegeln von $18.500 \mu \mathrm{g} / \mathrm{l})$.

- Zuchinali P et al. Short-term effects of high-dose caffeine on cardiac arrhythmias in patients with heart failure: a randomized clinical trial. JAMA Intern Med 2017, online 17. Oktober; doi: 10.1001/jamainternmed.2016.6374

\section{KOMMENTAR}

Der Zusammenhang zwischen Koffein und Herzrhythmusstörungen wird trotz zahlreicher Studien weiterhin kontrovers diskutiert. Der Koffeingehalt in einer Tasse Kaffee schwankt nach Volumen, Sorte und Zubereitung teils erheblich (im Mittel zwischen 40 und $120 \mathrm{mg}$ ). Die vorliegende Studie hat viele Vorzüge. Sie ist methodisch überzeugend und hat Patienten mit deutlicher Herzinsuffizienz untersucht. Die verabreichten Koffeindosen waren hoch, das Herz wurde kontinuierlich in Ruhe und auch bei Belastung überwacht. Es ist schon bemerkenswert, wenn hier keine negativen Folgen hoher Koffeindosen nachweisbar sind. Kritische Punkte sind die niedrige Probandenzahl und die Beschränkung auf Kurzzeiteffekte. Viele Ärzte erlauben ihren Herzpatienten zwei bis vier Tassen koffeinhaltigen Kaffee täglich. Wen das froh, frisch und glücklich macht, der kann dabei bleiben.

Prof. Dr. med. H. Holzgreve

\section{Eisenmangeldiagnose in einer Minute - ohne Blutabnahme}

Bei der Entstehung von Hämoglobin ist der Einbau eines Eisenatoms in das Protoporphyrin-IX-Molekül der letzte Schritt bei der Erzeugung von Häm. Steht nicht genügend Eisen zur Verfügung, wird stattdessen Zink eingebaut. Das entstandene Zink-Protoporphyrin bleibt für die gesamte Lebenszeit des Erythrozyten erhalten und kann daher als biochemischer Indikator für einen funktionellen Eisenmangel herangezogen werden.

Dieses Prinzip liegt einer neuen fluoreszenzfotometrischen In-vivo-Methode zugrunde, die im Laser-Forschungslabor am Klinikum der Universi-

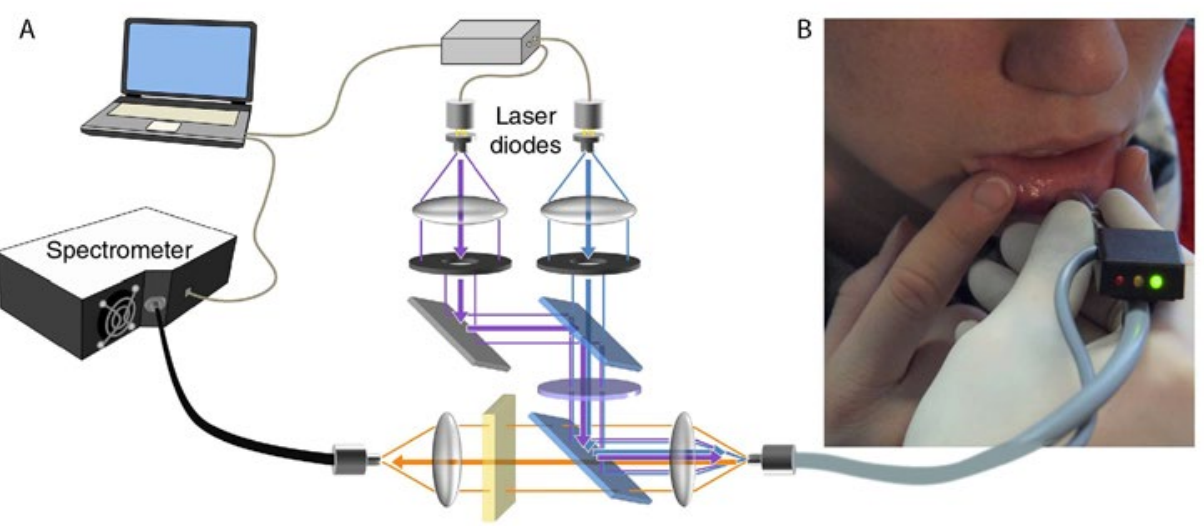

Gewebe-Fluoreszenzmessung unter Verwendung zweier verschiedener Wellenlängen. tät München entwickelt wurde. Eine Sonde, die Licht mit den definierten Wellenlängen 407 und $425 \mathrm{~nm}$ aussendet, wird an der Unterlippe an der Grenze zwischen Lippenrot und Mundschleimhaut aufgesetzt. Die Fluoreszenz von Protoporphyrin IX ist bei $407 \mathrm{~nm}$ größer als bei $425 \mathrm{~nm}$. Aus dieser Differenz lässt sich die Konzentration von Protoporphyrin IX errechnen, die ein indirektes Maß für den Eisenmangel darstellt.

Bei 56 Schwangeren wurde drei Tage nach der Geburt ein mittlerer Hämoglobinwert von 10,9 g/dl gemessen. Bei 35 dieser Frauen lag ein Eisenmangel vor. Beim Vergleich der mittels HPLC und der nicht-invasiv gemessenen Zink-ProtoporphyrinKonzentration ergab sich eine enge lineare Korrelation bei allen Frauen, ob mit oder ohne Eisenmangel. Sensitivität und Spezifität des nicht-invasiven Tests für einen Eisenmangel lagen bei $97 \%$ und $90 \%$. In einer Testreihe erwies sich die beschriebene Stelle als Messort mit der besten Korrelation. Demnächst ist geplant, das Verfahren auch bei Kindern zu versuchen, wo die Bedingungen aufgrund der Zartheit des Gewebes wahrscheinlich noch besser sind.

Prof. Dr. med. H. S. FüeßI

- Hennig Getal. (gmb31@)columbia.edu): Non-invasive detection of iron deficiency by fluorescence measurement of erythrocyte zinc protoporphyrin in the lip. Nature Communications 2016 online 17. Februar; doi: 10.1038/ncomms10776 